\title{
Experimental Studies on a Preferable Blade Profile for High Efficiency and the Blade Characteristics of Darrieus-Type Cross-Flow Water Turbines*
}

\author{
Yasuo TAKAMATSU**, Akinori FURUKAWA**, \\ Kusuo OKUMA** and Kazuki TAKENOUCHI***
}

\begin{abstract}
Fluid forces and moment acting on a blade of a Darrieus-type runner were measured in addition to the blade performance. The characteristics for various type of blades were evaluated from the measured data with theoretical considerations in an inviscid flow. Some features of those characteristics, including the dynamic effects caused by an unsteady relative flow around the Darrieus blade, are shown in the present paper. For high efficiency, a noncambered thin blade with relatively long chord length is recommended. Its characteristics are high lift slope, high lift at zero angle of attack and relatively low drag in a wide no-stall region of attack angles. It should also be set tangent to the runner pitch-circle at the $50 \%$ chord point of the blade.
\end{abstract}

Key Words: Hydraulic Machine, Water Turbine, Cross-Flow, Darrieus Runner, Blade Performance, Blade Profile, Blade Setting Attitude

\section{Introduction}

In order to utilize undeveloped natural extralowhead water power or to recover wasted water power in the industry for a diversification of energy resources as a means of energy conservation, some trials have already been made with Darrieus-type crossflow water turbines, and the feasibility of this kind of water turbine for practical use is being proved ${ }^{(1)}$. However, a different type of blade section, as symmetrical sections NACA0012(2), NACA64 ${ }_{3}-021^{(3)}$ or a circular cambered section with the same curvature as the runner pitch circle NACA63 $-018^{(4)}$, has been tested for the runner blade in each of those experiments. Experimental results on the runner performance with various geometrical attitudes of the blade have also been presented ${ }^{(2),(5)}$. Guiding principles of the blade section and the blade setting attitude for designing a high-performance runner have not been made clear

- Received 19th November, 1990. Paper No.89-1004A

** Department of Mechanical Engineering, Kyushu University, 6-10-1, Hakozaki, Higashi-ku, Fukuoka 812, Japan

*** Department of Industrial Design, Kyushu Institute of Design, 4-9-1, Shiobaru, Minami-ku, Fukuoka 815, Japan yet. On the other hand, some theoretical studies on these effects have also been carried out ${ }^{(6)}$. However, in their calculations, characteristics of the Darrieus blade in a circular motion are different from those of the blade in a linear motion in a uniform flow ${ }^{(7),(8)}$, and wind-tunnel-tested data for a stationary blade in a uniform flow were used themselves to estimate the characteristics of the runner blade without taking into account differences in their motion.

Fundamental differences in blade characteristics between a Darrieus blade in a circular motion and a stationary one in a uniform flow have been described by use of an inviscid flow analysis in the preceding paper $^{(9)}$. In a real flow, such viscous effects as dynamic stall are, thereupon, exerted on the Darrieus blade characteristics, so instantaneous fluid forces and moment acting on a blade of the two-dimensional Darrieus runner in operation have been measured in order to evaluate these in a real flow.

In the present paper, measured fluid forces and moment are illustrated, and then the Darrieus blade characteristics, as lift coefficient, drag coefficient and so on against the attack angle, are evaluated from those data in accordance with theoretical considera. tions ${ }^{(9)}$. By comparing calculated blade efficiency by use of measured blade characteristics with the directly measured blade efficiency, the validity of the 
proposed method ${ }^{(9)}$ to obtain the characteristics of an equivalent stationary blade in a linear motion corresponding to those of the Darrieus blade is examined. A universal approach to selecting a blade section and a blade setting attitude for high runner performance is discussed.

\section{Experimental Apparatus}

Figure 1 shows a schematic view of the experimental apparatus. Water, supplied to the upstream open channel, flows to the downstream water pool through the test section (1) of rectangular cross section $B \times S=200 \mathrm{~mm} \times 400 \mathrm{~mm}$. The flow rate $Q$ is measured by an orifice mounted on the delivery pipe of the water supplying pump. The amount of available head imposed on the runner $H$ is adjusted by manual control of a butterfly valve (6) set in the draft tube, and evaluated by subtraction of head loss between the upstream open channel and the downstream section of the runner in a state without runner blade from the difference in averaged total head between these sections in the operating state. A single-bladed, twodimensional Darrieus runner with the radius $R$ of the runner pitch circle is composed of a shaft perpendicular to the flow, a blade (7) with a straight $\operatorname{span} B$, supporting arms (8) and end disks (9) as shown in Fig. 1 , and it is placed in the test section (1) vertically.

Figure 2 shows the sectional profiles of seven kinds of tested blades. Five kinds (blades A, B, C, D, E) are adopted from NACA 4-digit series aerofoils of different thickness ratios, maximum cambers and chord lengths. The radius of the camber line of two circular cambered sections, A and D, is the same as the runner pitch circle. The other two have special blade shapes; one is designed by curving the chord line of a blade, having an almost fixed center of pressure in a uniform flow, along the runner pitch circle (blade F), and the other has low-drag characteristics in a uniform flow (blade $G$ ). The tested blades are fixed to the end of supporting arms at the quarter chord point


Fig. 1 Experimental apparatus of a symmetrical blade or at the point on a camber line corresponding to the quarter chord point of a cambered blade.

The fluid forces and moment acting on a runner blade are measured with three strain gauges (10) pasted on each supporting arm, as shown in Fig. 3. The output signals from strain gauges are picked up from a rotating runner through a slip ring (2) and recorded after analog-digital conversion. A time-variation in output signals in one revolution of runner is averaged over 16 revolutions. Instantaneous magnitudes of radial and tangential components, $F_{r}$ and $F_{u}$, of fluid force and moment $M$ at the supporting point of blade are calculated with calibration curves between stress and strain from the averaged time variation of output signals by subtraction of magnitudes of the centrifu. gal force acting on the blade in air, and the centrifugal force and fluid force acting on the supporting arms in water.

The experiments were carried out with $\bar{V}=1.0$ $\mathrm{m} / \mathrm{s}$ over the wide range of speed ratio $U / \bar{V}$ and blade setting angle $\delta$, where $U$ is the peripheral speed of the runner on the pitch circle and $\bar{V}$ is the cross-sectionally averaged incoming absolute velocity. $\delta$ is taken as $0^{\circ}$ when the chord line of the blade is tangent to a pitch circle at the quarter chord point for symmetrical blades and when the camber line is just laid down on the pitch circle for cambered blades as shown in Fig. 2 and defined as positive when the leading edge of the blade is radislly inside of the position of $\delta=0^{\circ}$.

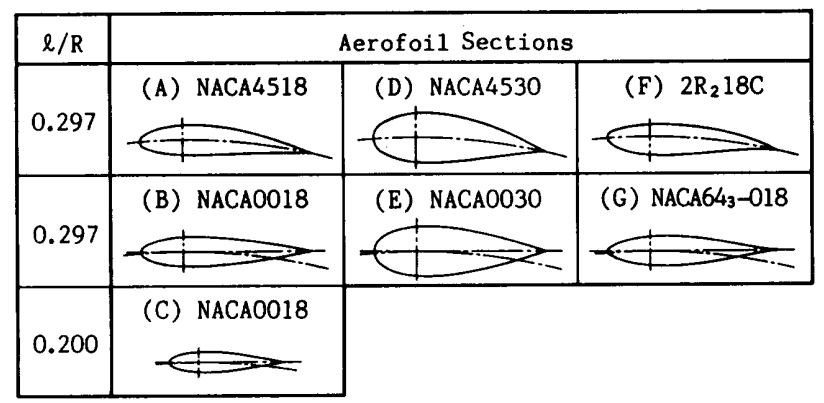

Fig. 2 Sectional profiles of tested blades

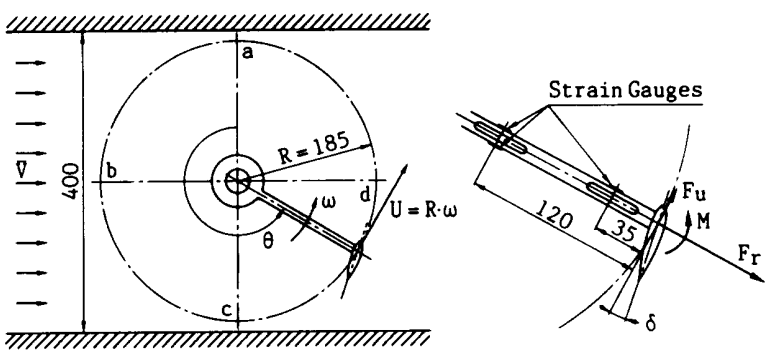

Fig. 3 Forces and moment acting on blade and crosssectional view of test section 


\section{Experimental Results}

\section{1 Blade efficiency of tested sections}

Measured blade efficiency $\eta_{b}$ is defined by the next equation, as a ratio of generated power averaged over a revolution to water power determined by a product of the weight-flow rate and the available head imposed on the runner,

$$
\eta_{b}=\left[\left(\bar{F}_{u} \cdot R+\bar{M}\right) \omega\right] /(\rho g Q H)
$$

where denotes values averaged over a revolution of the runner, $\omega$ is the angular velocity of rotation, $\rho$ is the density of water, and $g$ is the gravitational acceleration.

Figure 4 shows variations of $\eta_{b}$ of each tested section against $U / \bar{V}$ or $\delta$, where one of $U / \bar{V}$ and $\delta$ is changed from the values at the operating condition of maximum blade efficiency, and the other is fixed to that value. The maximum value of $\eta_{b}$ is obtained when the camber line is just laid down on the runner pitch circle, $\delta=0^{\circ}$, for the cambered section and when $\delta$ takes a negative value in the range from $-5^{\circ}$ to $-2.5^{\circ}$ for the symmetrical section though the value of $\delta$ is dependent on the blade thickness or chord length. On the whole, a blade with longer chord length and smaller thickness brings about a higher blade efficiency, while the difference of blade camber may not influence the speed ratio $U / \bar{V}$ with the maximum values of $\eta_{b}$. The best efficiency, $\eta_{b}=0.62$, was obtained by blade $\mathrm{B}$ among all tested sections, meaning that a symmetrical section with small thickness and long chord length brings about better blade efficiencies. Advantages of the low drag section (blade G) are not found, the efficiencies obtained by $2 R_{2} 18 C$ (blade F) are relatively high, and the variation in $\eta_{b}$ against $U / \bar{V}$ is less than those of the other sections.

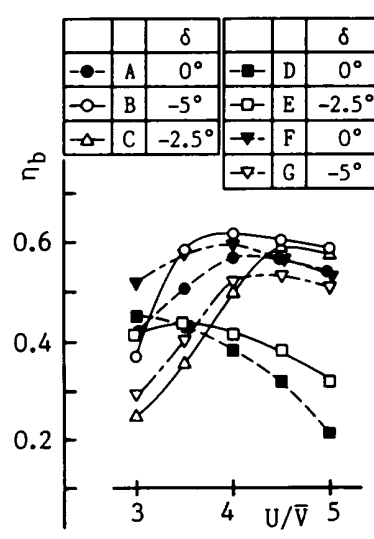

(a) Dependency on $U / \bar{V}$

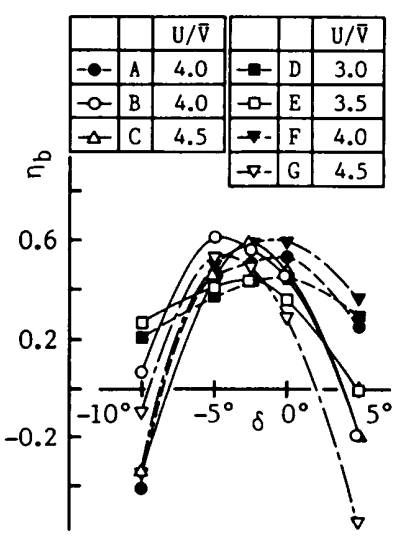

(b) Dependency on $\delta$
Fig. 4 Change of measured blade efficiency with blade profile and setting angle

\subsection{Evaluation of measured Darrieus blade char- acteristics}

Equation (2) is often employed for predicting blade efficiency $\eta_{c}$ in the estimation of runner performance $^{(2),(5)}$ :

$$
\eta_{c}=\frac{\int_{0}^{2}\left(F_{u} \cdot R+M\right) \omega d \theta}{\int_{0}^{2 \pi}\left[\left(F_{u} \cdot R+M\right) \omega+\frac{1}{2} \rho W^{3} B l C_{d}\right] d \theta}
$$

where the denominator expresses the total water power supplied to a blade of the runner in one revolution as the sum of an instantaneous generated power and a power loss consumed to maintain a flow against a drag of the blade. In order to discuss the difference in blade efficiency described in the preceding section by use of Eq. ( 2 ) from the aspect of the blade characteristics of the Darrieus blade, it is necessary to obtain Darrieus blade characteristics of each tested section from measured data of fluid forces in a real flow.

The direction of action of lift force $L$ (lift coefficient $C_{l}$ ) and drag force $D$ (drag coefficient $C_{d}$ ) of the Darrieus blade is obtained from the local attack angle at the center of pressure $\mathrm{e}^{(9)}$. The distance $x_{c}$ between the leading edge and the center of pressure is determined with measured data of $F_{r}, F_{u}$ and $M$ by the next equation.

$$
\begin{aligned}
& x_{c} / l=0.25+(M-\Delta M) /\left\{\left[\left(F_{r}-\Delta F_{r}\right) \cos \left(\delta-\delta_{0}\right)\right.\right. \\
& \left.\left.\quad+\left(F_{u}-\Delta F_{u}\right) \sin \left(\delta-\delta_{0}\right)\right] l\right\}
\end{aligned}
$$

Figure 5 illustrates a geometrical relation between relative flow, blade forces and blade attitude. The relations between $L, D$ and $F_{r}, F_{u}$ acting on the cambered blade are expressed by the next equations with the angle of direction of action of lift force $\delta_{c}$ [Eq. (31) in Ref. (9)], instead of the local attack angle at the center of pressure, in the same manner proposed in the preceding paper ${ }^{(9)}$.

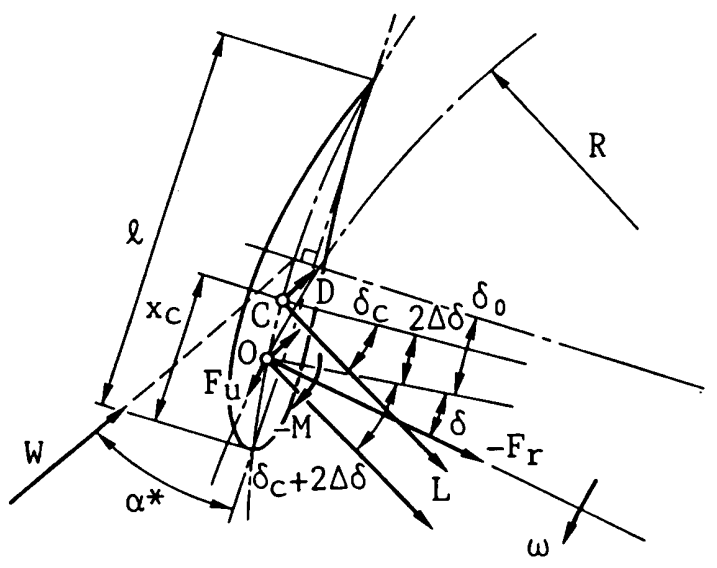

Fig. 5 Geometrical relations between relative flow and fluid forces acting on Darrieus blade ( $\mathrm{O}$ : supporting point, $\mathrm{C}:$ center of pressure) 


$$
\begin{gathered}
F_{r}=-0.5 \rho W^{2} B l\left[C_{\iota} \cos \left(\delta_{c}-\delta+2 \Delta \delta\right)\right. \\
\left.\quad+C_{d} \sin \left(\delta_{c}-\delta+2 \Delta \delta\right)\right]+\Delta F_{r} \\
F_{u}=0.5 \rho W^{2} B l\left[C_{\iota} \sin \left(\delta_{c}-\delta+2 \Delta \delta\right)\right. \\
\left.\quad-C_{d} \cos \left(\delta_{c}-\delta+2 \Delta \delta\right)\right]+\Delta F_{u}
\end{gathered}
$$

where $\Delta \delta$ and $\delta_{0}$ are the angles shown in Fig. 5 and calculated with the relation of $x_{c}$ and blade camber, and $\Delta F_{r}, \Delta F_{u}$ and $\Delta M$ are the components of additional force and moment of centrifugal force and Coriolis force caused by a circular motion of a blade and calculated beforehand by the blade profile and setting attitude.

A procedure in the calculation to evaluate the lift and drag coefficients of the Darrieus blade $C_{l}, C_{d}$ is as follows. At first, $x_{c}$ is calculated with the measured blade forces and moment at every rotating position $\theta$. And next, a direction angle of lift force $\delta_{c}$ is given by using instantaneous relative flow (relative flow velocity $W$ and attack angle $\alpha^{*}$ ) evaluated in the flow calculation ${ }^{(5)}$ with consideration of the existence of the walls of flow passage and vortex street shed from the blade. $C_{l}$ and $C_{d}$ are determined with the above values of $W$ and $\delta_{c}$ by solving Eqs. (4) and (5) simultaneously. This iterative procedure is continued until convergence of time variation in the amount of circulation around the Darrieus blade in one revolution is achieved. Figure 6 shows examples of the results given in the calculation according to the above procedure. The center of pressure $x_{c} / l$ [shown in Fig. $6(d)]$ is, at first, calculated with the measured data, $C_{r}, C_{u}$ and $C_{m}$ [Figs. 6(a), (b) and (c)] . Relative flow [Figs. 6(e) and (f)] and blade characteristics [Figs. $6(\mathrm{~g})$ and $(\mathrm{h})$ ] are secondly evaluated by the iterative calculation.

Results of a cambered section (blade A) and a symmetrical section (blade B) are compared in Fig. 6 . For symmetrical blade B, a moment acting at the supporting point, a quarter chord point of blade, always takes a negative value, and the magnitudes of tangential and radial forces are larger in the upstream half of a rotation than in the downstream half. On the other hand, for cambered blade A, a moment at the supporting point takes a value close to zero over a revolution of the runner, and the differences in the magnitudes of tangential force and radial force between the upstream half and the downstream half are less obvious than for blade $B$ except for in a range near $\theta=3 \pi / 2$, where an incoming flow to a blade might be deformed by the existence of wake formed by a runner shaft and the effect of the blade in the upstream half of a rotation. The relative flow to the blade is almost the same between both blades, as shown in Figs. 6(e) and ( $\mathrm{f}$ ). The above differences in measured data bring about different blade characteristics in Figs. $6(\mathrm{~g})$ and $(\mathrm{h})$ caused by the exis-

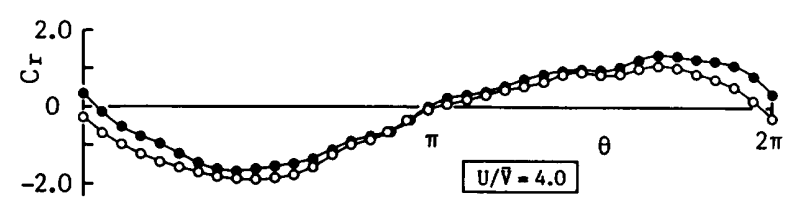

(a) Radial force $\left[C_{r}=F_{r} /\left(2 \rho \bar{V}^{2} B R\right)\right]$

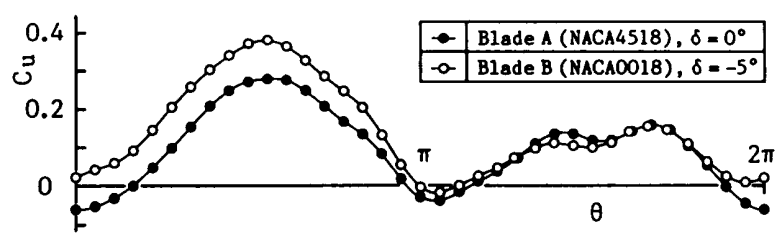

(b) Tangential force $\left[C_{u}=F_{u} /\left(2 \rho \bar{V}^{2} B R\right)\right]$

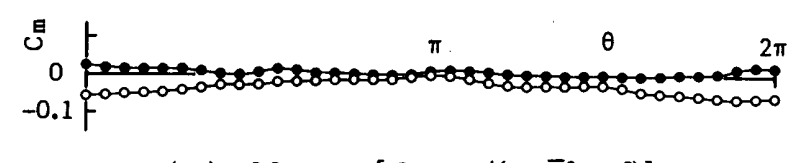

(c) Moment $\left[C_{m}=M /\left(2 \rho \bar{V}^{2} B R^{2}\right)\right]$

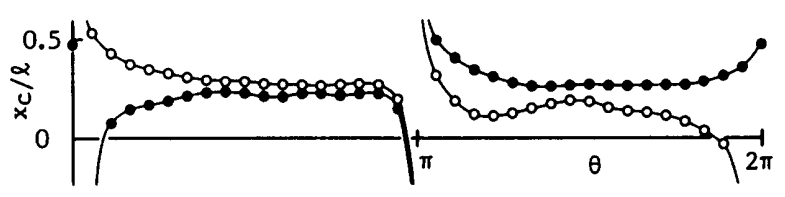

(d) Center of pressure $\left(x_{c} / l\right)$

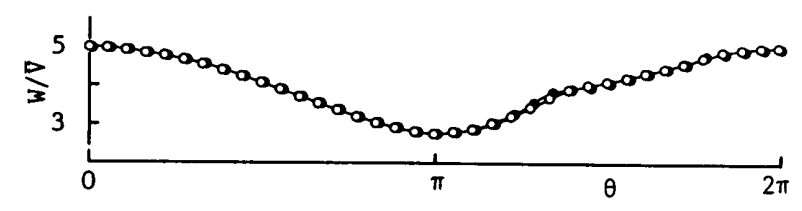

(e) Relative velocity $(W)$

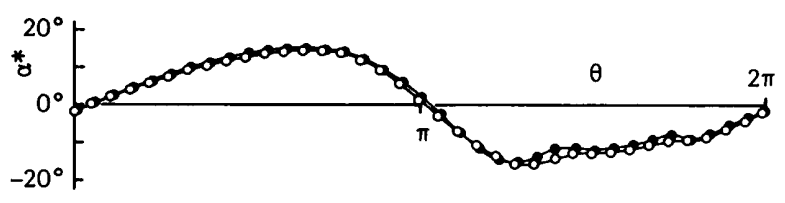

(f) Attack angle $\left(\alpha^{*}\right)$

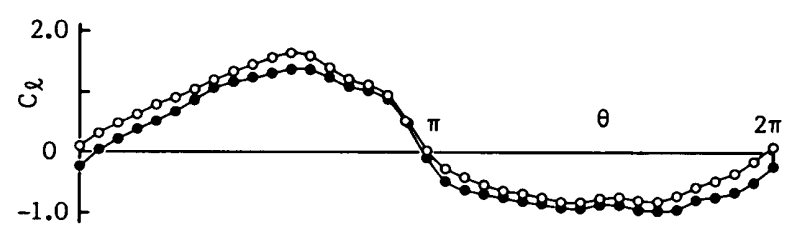

(g) Lift coefficient $\left(C_{\imath}\right)$

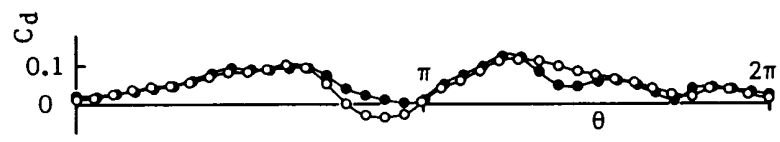

(h) Drag coefficient $\left(C_{d}\right)$

Fig. 6 Results of measurement of fluid forces and evaluation of Darrieus blade characteristics 
tence of the camber of the blade.

\section{3 Verification of Darrieus blade characteris- tics}

The values of blade efficiency $\eta_{c}$ are calculated in a wide range of operating conditions of $U / \bar{V}$ and $\delta$ by substituting the evaluated Darrieus blade characteristics into Eq. (2). The results are compared in Fig. 7 with the measured blade efficiency $\eta_{b}$ obtained by Eq. (1). Also shown by dotted line in Fig. 7(a) are the results obtained by use of blade characteristics evaluated in the ordinary manner ${ }^{(2),(7)}$, in which it is assumed that lift force acts orthogonally to the relative flow at the supporting point of the blade or $50 \%$ chord point of the blade. These results obtained in the ordinary manner give a greater discrepancy from $\eta_{b}$ in a higher range of $U / \bar{V}$. On the other hand, when
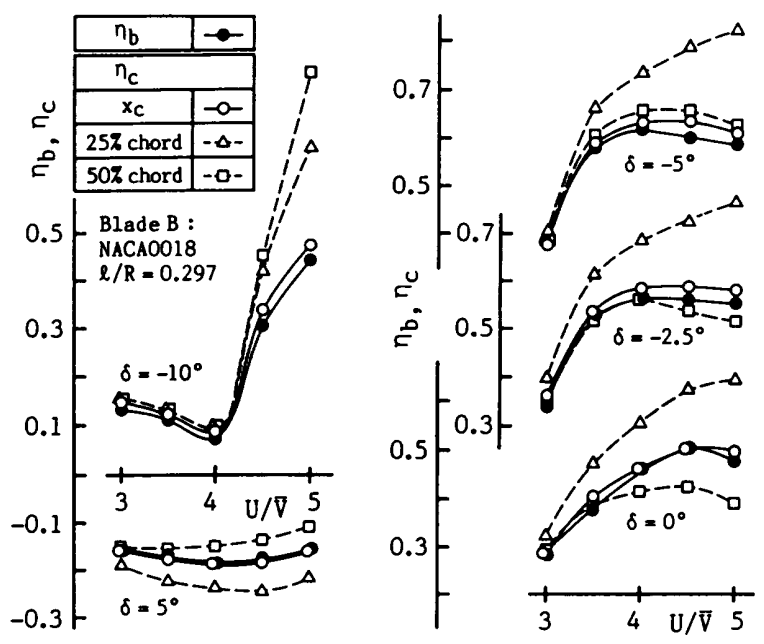

(a) Dependency on representative point for flow calcula. tion
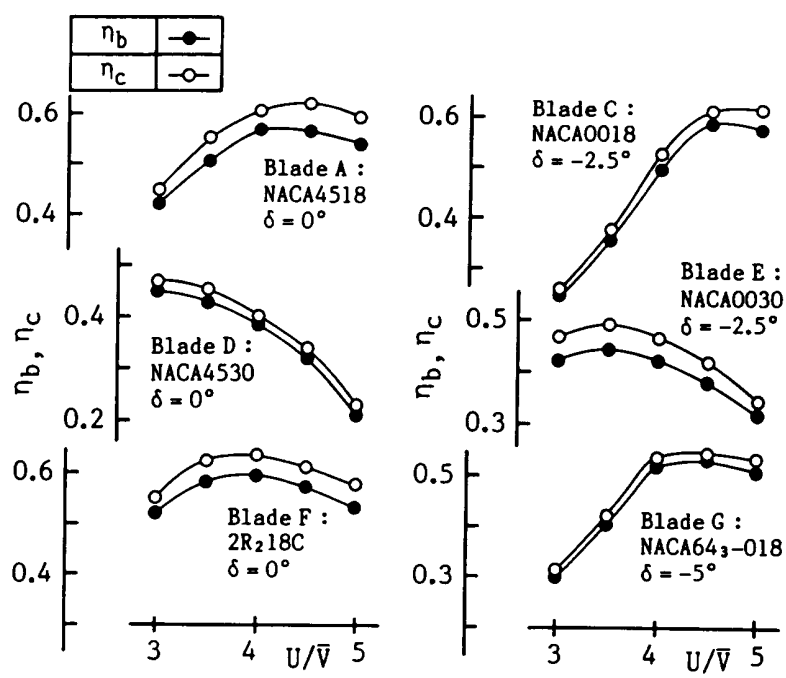

(b) Dependency on blade profile

Fig. 7 Comparison of $\eta_{c}$ calculated by Eq. (2) with measured blade efficiency $\eta_{0}$ blade characteristics are evaluated with the theoretical consideration ${ }^{(9)}$ of the directions of lift and drag forces, values of $\eta_{c}$ can predict that of $\eta_{b}$ with adequate accuracy over wide ranges of $U / \bar{V}$ and $\delta$ for blade $B$ as found in Fig. 7(a) and over a wide range of $U / \bar{V}$ for other blades in Fig. 7(b). Predicted values of $\eta_{c}$ are somewhat higher than measured ones of $\eta_{b}$ since a measured value of available head $H$ in calculation of $\eta_{b}$ contains a mixing loss of deformed flow by runner blade to become a uniform flow. Consequently, the relatively close agreement of $\eta_{c}$ with $\eta_{b}$ supports the validity of the consideration of the direction of blade force and relative flow in addition to the reliability of evaluated blade characteristics of Darrieus blade in the authors' manner from measured forces.

\subsection{Comparison of Darrieus blade characteris- tics for various blade sections}

Figure 8 shows blade characteristics of Darrieus blade against equivalent attack angle $\alpha^{*}$ for all tested sections. Solid lines with white circles indicate the variation of blade characteristics of each tested section in a revolution of runner at the operating condition with the maximum value of $\eta_{b}$. The additional letters $a, b, c$ and $d$ laid by the side of the white circle correspond to the rotating positions of blade $\theta=0$, $\pi / 2, \pi, 3 \pi / 2$, respectively, as shown in Fig. 3 . Symbols of + or $x$ indicate instantaneous values in some operating conditions when one of $U / \bar{V}$ or $\delta$ is altered from the optimum value at which the maximum $\eta_{b}$ is obtained and the other is fixed to the optimum value. It is found from the results that blade characteristics of $C_{l}, C_{d}$ and $x_{c} / l$ have a tendency to vary roughly along a line for each tested section in an advancing side of blade rotation from the position $d$ to $b$ through a. Broken lines in this figure express the next formulas approximating lift and drag coefficients in this rotating region against $\alpha^{*}$ :

$$
\begin{aligned}
& C_{l}=k_{l} \sin \alpha^{*}+C_{l 0} \\
& C_{d}=k_{d} \alpha^{* 2}+C_{d 0}
\end{aligned}
$$

where the values of parameters $k_{l}, C_{l 0}, k_{d}$ and $C_{d 0}$ for each blade section are listed in Table 1.

By comparing blade characteristics in this region of various blade sections, the following several features are revealed. A difference of blade camber strongly affects the value of $C_{l 0}$, which is the lift coefficient at $\alpha^{*}=0$, and the variation of $x_{c} / l$ against $\alpha^{*}$. That is, a Darrieus blade with a circular camber with the same radius of the runner pitch circle almost corresponds to a noncambered blade at rest in a uniform flow and a symmetrical Darrieus one corresponds to a cambered one in a uniform flow. Tendencies similar qualitatively to those of a blade at rest in a uniform steady flow are observed; that is, a thick 
blade has less lift slope, $d C_{l} / d \alpha^{*}$, and larger drag coefficient at $\alpha^{*}=0^{\circ}, C_{d 0}$. On the other hand, in a retreating side of blade rotation from the position $b$ to $\mathrm{d}$ through $\mathrm{c}$, hysteresis arises in blade characteristics due to the unsteady effect of relative flow to a blade. The feature of the dynamic behavior appears distinct-

Table 1 Fundamental parameters for blade characteristics of tested Darrieus blade

\begin{tabular}{|c|c|r|r|r|r|r|r|}
\hline $\begin{array}{c}\text { Tested } \\
\text { Section }\end{array}$ & A & B & C & D & E & F & G \\
\hline$k_{\ell}$ & 5.40 & 5.40 & 5.40 & 3.41 & 3.87 & 5.40 & 5.40 \\
\hline$C_{\ell_{0}}$ & -0.07 & 0.30 & 0.21 & -0.06 & 0.27 & -0.04 & 0.30 \\
\hline$k_{d}$ & 1.27 & 1.27 & 1.27 & 0.78 & 1.02 & 1.27 & 1.50 \\
\hline$C_{d o}$ & 0.018 & 0.018 & 0.018 & 0.038 & 0.030 & 0.018 & 0.021 \\
\hline$\alpha_{\text {su }}^{*}$ & $17^{\circ}$ & $20^{\circ}$ & $15^{\circ}$ & $21^{\circ}$ & $23^{\circ}$ & $20^{\circ}$ & $18^{\circ}$ \\
\hline$\alpha_{\text {sd }}^{*}$ & $-18^{\circ}$ & $-16^{\circ}$ & $-13^{\circ}$ & $-13^{\circ}$ & $-19^{\circ}$ & $-18^{\circ}$ & $-15^{\circ}$ \\
\hline
\end{tabular}

ly in the upstream half of blade rotation. When a blade is in the stalled state, a great increase in $C_{d}$ and a shift of $x_{c}$ toward the trailing edge of the blade begin first. In this case, $C_{l}$ continues to increase linearly even after $C_{d}$ begins to increase, and a sudden collapse of $C_{l}$ follows it; that is the typical feature of the dynamic stall. On the contrary, when a blade is in the unstalled state, $C_{l}$ varies roughly along a straight line while $C_{d}$ shows a peculiar behavior which cannot be observed in the stationary state, that is, a sudden decrease in the vicinity of the maximum $\alpha^{*}$, and in some cases $C_{d}$ takes a negative value locally.

In the stalled state of a Darrieus blade, $C_{d}$ increases greatly with absolute value of $\alpha^{*},\left|\alpha^{*}\right|$ and takes a maximum value $C_{d m}$ in the vicinity of the maximum of $\left|\alpha^{*}\right|$. While $C_{d m}$ increases with maximum $\left|\alpha^{*}\right|$, the behavior of $C_{d}$ approaching $C_{d m}$ in a revolu-
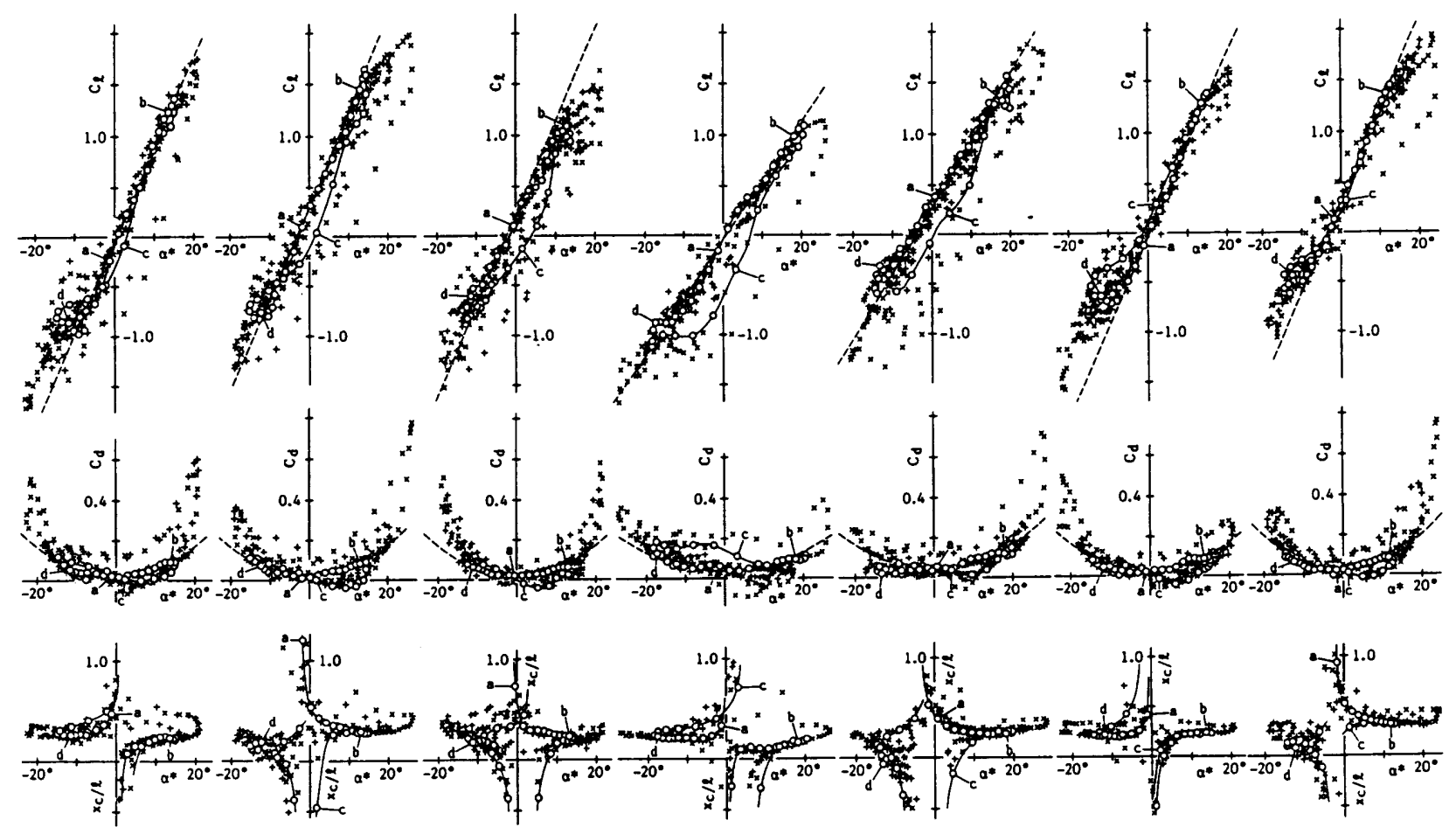
(a) Blade A
(b) Blade B
(c) Blade C
(d) Blade D
(e) Blade E
(f) Blade F
(g) Blade G

Fig. 8 Darrieus blade characteristics of tested sections ( $O$ : at maximum efficiency, +: dependency on $U / \bar{V}, \times:$ dependency on $\delta$ )

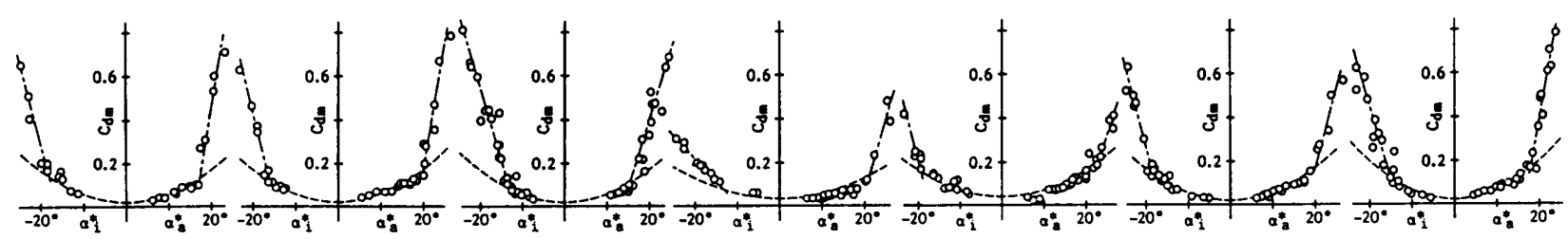
(a) Blade A
(b) Blade B
(c) Blade C
(d) Blade D
(e) Blade $\mathrm{E}$
(f) Blade F
(g) Blade G

Fig. 9 Maximum of drag coefficient in wide operating conditions $\left[O: C_{d m},---:\right.$ Eq. $\left.(7)\right]$ 
tion of the runner is not identical for a different value of maximum $\left|\alpha^{*}\right|$, which is denoted as $\alpha_{a}^{*}$ in the upstream half and $\alpha_{i}^{*}$ in the downstream half of blade rotation. Then, the relations between $C_{d m}$ and $\alpha_{a}^{*}, \alpha_{i}^{*}$ in each operating condition are plotted in Fig. 9 as the drag characteristics of Darrieus blade in the stalled state. Values of $C_{d m}$ apart from the broken line, which is an approximate line of drag coefficient in the unstalled state expressed by Eq. 7 ), are found in Fig. 9. By considering those values as the maximum drag coefficient caused by stall, the stall angles of attack of Darrieus blade, $\alpha_{s u}^{*}$ in the upstream half and $\alpha_{s d}^{*}$ in the downstream half, are obtained as the values at the intersection of a dot-dash line through the above $C_{d m}$ and a broken line. Also listed in Table 1 are the obtained stall angles of Darrieus blade for tested sections. From these values, some qualitative features are found on a blade section; that is, $\left|\alpha_{s d}^{*}\right|<\left|\alpha_{s u}^{*}\right|$ for a symmetrical section, $\left|\alpha_{s d}^{*}\right| \cong\left|\alpha_{s u}^{*}\right|$ for a cambered section, and a thick blade with long chord length has wide no-stall region of $\alpha^{*}$.

\section{Blade Section and Attitude for High Runner Performance}

It is clear that the differences in the values of maximum $\eta_{b}$ themselves and operating conditions $U / \bar{V}$ and $\delta$ at maximum $\eta_{b}$ between tested sections arise from the differences in blade characteristics. To obtain guiding principles for high efficiency, therefore, it is necessary to explain important factors on blade efficiency by considering a quantitative relation between blade efficiency and blade characteristics. The effects of the value of parameters $k_{l}, C_{l 0}, k_{d}$ and $C_{d 0}$ on blade efficiency are examined by use of an estimation $^{(9)}$ of blade efficiency assuming that Darrieus blade characteristics can be expressed with Eqs. (6) and ( 7), since blade characteristics at an optimum operating condition are in a no-stall region of $\alpha^{*}$ and have relatively small hysteresis.

Figure 10 shows the results in the terms of coefficients of generated power, $C_{p o}=C_{p o l}+C_{p o d}$ and coefficient of power consumed by blade drag $C_{p d l}$ in Eq. ( 8 ), calculated by altering values of parameters from the values of blade $B$ in Table 1. The power coefficient is defined as a ratio of averaged power over a revolution of runner to $\left(\rho \bar{V}^{3} B R\right)$, and the components of power generated by lift force and drag force of blade are defined as $C_{p o t}$ and $C_{p o d}$, respectively. That is, $\eta_{c}$ is expressed with these power coefficients by the next equation.

$$
\eta_{c}=\left(C_{p o l}+C_{p o d}\right) /\left(C_{p o}+C_{p d l}\right)
$$

The broken line in this figure shows the results obtained with the values of the parameter for blade B. Other lines are obtained by altering a value of one parameter among others from the value for blade B in Table 1 to a listed one in the figure.

The results in Fig. 10 reveal the following facts. It is, at first, found from considering the dependency of $\eta_{c}$ on $U / \bar{V}$ that both $-1.0 \times C_{p o d}$ and $C_{p d l}$ increase with $U / \bar{V}$ together while $C_{p o l}$ also increases so that the blade efficiency becomes higher as $U / \bar{V}$ is lowered. On the other hand, in the region of low $U / \bar{V}$, a great increase of $C_{d}$, caused by stall as shown in Fig. 8, deteriorates blade efficiency since the blade experiences a wide range of attack angle in a revolution of the runner. This is the reason that the values of $U / \bar{V}$ with the maximum efficiency in the cases of the thick blades $\mathrm{D}$ and $\mathrm{E}$, the blade characteristics of which have wide no-stall region of attack angle as shown in Fig. 9, become lower than the other blades, as shown in Fig. 4. It is secondly seen from considering the effects of $\delta$ that while $C_{p o t}$ slightly increases with $\delta$, since $\alpha^{*}$ increases during the upstream half of blade rotation followed by Fig. 6 ( $f$ ), where the effects of deflection of incoming flow on blade are less than in the downstream, the values of $-1.0 \times C_{p o d}$ and $C_{p d t}$ take the minimum in the vicinity of $\delta=-5^{\circ}$, so that the maximum of blade efficiency is obtained at this setting angle. This result indicates a similar tendency to that of the experimental result in Fig. 4 and suggests that $\delta$ should be selected as $\alpha^{*}$ varies within a no-stall region widely and with the center of the no-
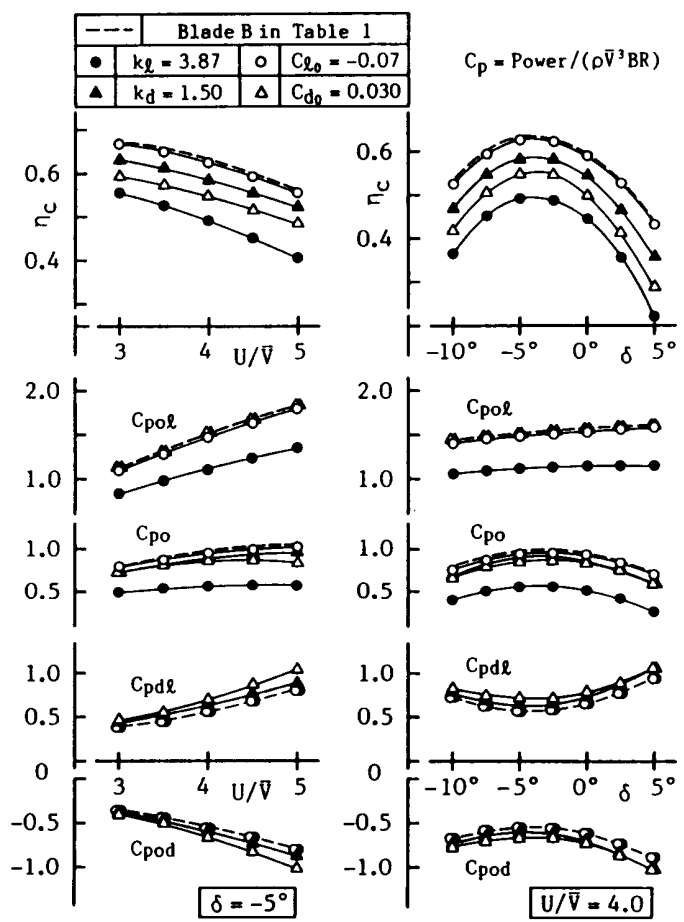

(a) Dependency on $U / \bar{V}$

(b) Dependency on $\delta$

Fig. 10 Change of blade efficiency and power coefficients with values of $k_{l}, C_{l 0}, k_{d}$ and $C_{d o}$ 
stall region as the average of $\alpha^{*}$ in a revolution. Since $C_{d}$ takes the minimum in the vicinity of $\alpha^{*}=0^{\circ}$ for all tested blades as shown in Fig. 8, and $\alpha^{*}$ is physically equivalent to a local attack angle at the midchord point $^{(9)}$, it is consequently considered that the most preferable blade setting attitude is for the blade to be set as tangent to the runner pitch circle at the midchord point of the blade.

The value of each parameter has a notable effect on the value of the blade efficiency itself, though little on the values of speed ratio $U / \bar{V}$ and blade setting angle $\delta$ where the maximum efficiency is obtained. Its tendency is made clear from Fig. 10 ; that is, a blade with larger values of $k_{l}$ and $C_{l 0}$ and smaller values of $k_{d}$ and $C_{d 0}$ gives a higher efficiency. In addition to that, the maximum efficiency of blade with wider nostall region of $\alpha^{*}$ becomes higher, as described previously. When the measured blade efficiency for all tested sections is considered from the aspect of blade characteristics according to the above results, the small $k_{\iota}$ and large $C_{d 0}$ of blades $\mathrm{D}$ and $\mathrm{E}$, narrow nostall region of $\alpha^{*}$ of blade $\mathrm{C}$, and large $k_{d}$ and narrow no-stall region of blade $\mathrm{G}$ deteriorate in blade efficiency. The higher value of $C_{l o}$ of blade $\mathrm{B}$ is considered to contribute to giving the best efficiency, while the values of other parameters are the same among blade $\mathrm{A}, \mathrm{B}$ and $\mathrm{F}$, because high $C_{l o}$ means that a greater proportion of power can be generated in the upstream half of blade rotation where deflection of incoming flow is less than in the downstream half.

\section{Conclusions}

The blade characteristics of a Darrieus-type runner for various blade sections were evaluated from the measured fluid forces and moment acting on a blade of the Darrieus runner. From the results, features of blade characteristics of the Darrieus blade and the effects of blade section on runner performance were discussed. The results are summarized as follows: (1) Blade characteristics of a Darrieus blade (a blade in a circular motion in a uniform flow) in a real flow were obtained in the manner proposed in the preceding paper. From the results, it was made clear that a noncambered and a circular cambered Darrieus blade correspond to a cambered and noncambered blade at rest in a uniform flow, respectively. It was also clarified that hysteresis appears in blade characteristics in a revolution of the runner, and a peculiar behavior of a sudden decrease of $C_{d}$ to a negative value occurs in the unstalled state.

(2) The validity of the expression of blade efficiency with blade characteristics and the idea on the direction of fluid force acting on a Darrieus blade were verified, and the reliability of blade characteristics of a Darrieus blade was confirmed from a close agreement between the evaluated blade efficiency with those characteristics and the one directly measured by the experiment.

(3) Guiding principles of blade section and blade setting attitude are proposed. A noncambered thin blade with relatively long chord length is recommended, and it should also be set tangent to the runner pitch circle at the $50 \%$ chord point of the blade.

This research was financially supported by the Grant-in-Aid from the Ministry of Education, Science and Culture, Japan, in 1984, 1985 and 1990.

\section{References}

(1) Takamatsu, Y., Furukawa, A. and Okuma, K., Research and Development of Darrieus-Type Water Turbine, J. Turbomachinery, (in Japanese), Vol. 13, No. 5(1985), p. 271.

(2) Miyake, Y., Koike, K., Tsugawa, T. and Murata, S., Performance Characteristics of High-SpeedType Cross Flow Turbine, Bull. Jpn. Soc. Mech. Eng., Vol. 27, No. 229(1984), p. 1446.

(3) Faure, T. D., Experimental Results of a Darrieus Type Vertical Axis Rotor in a Water Current, NRC Can., Div. Mech. Eng., TR-HY-005(1984).

(4) Kusunoki, R., Kotajima, Y., Kigata, Y., Miyagi, H. and Kojima, I., Power Generation of Tidal Current by Darrieus-Type Water turbine, Prepr. of Conf. Inst. Elec. Eng., (in Japanese), Vol. 11 (1984), p. 1382.

(5) Takamatsu, Y. Furukawa A., Okuma, K. and Takenouchi, K., Effects of Geometrical Attitude of Blade on Hydrodynamic Performance of Darrieus-Type Cross-Flow Water Turbine, Bull. Jpn. Soc. Mech. Eng., Vol. 28, No. 240(1985), p. 1092.

(6) Murata, S., Miyake, Y. and Tsugawa, T., Research and Development of Darrieus-Type Water Turbine, Bull. Jpn. Soc. Mech. Eng., Vol. 26, No. 211(1983), p. 43.

(7) Takamatsu, Y., Furukawa, A., Okuma, K. and Shimogama, Y., Study on Hydrodynamic Performance of Darrieus-Type Cross-Flow Water Turbine, Bull. Jpn. Soc. Mech. Eng., Vol. 28, No. 240 (1985), p. 1119.

(8) Wilson, R. E., Lissaman, P. D. S., James, M. and Mckie, W. R., Aerodynamic Loads on a Darrieus Rotor Blade, Trans. Am. Soc. Mech. Eng., Ser.I, Vol. 105, No. 1(1983), p. 53.

(9) Furukawa, A., Takamatsu, Y. and Takenouchi, K., Theoretical Considerations in an Approximate Method for Estimating the Blade Performance of a Darrieus-Type Cross-Flow Water Turbine, Mem. Fac. Eng., Kyushu Univ., Vol. 50, No. 1 (1990), p. 1. 